\title{
Women with prior gestational diabetes mellitus and prediabetes are characterised by a decreased incretin effect
}

\author{
Signe Foghsgaard ${ }^{1,2,3} \cdot$ Louise Vedtofte $^{1}$ - Camilla Andreasen ${ }^{1}$ Emilie S. Andersen ${ }^{1}$. \\ Emilie Bahne $^{1}$ - Jonatan I. Bagger ${ }^{1}$ • Jens A. Svare ${ }^{4}$. Jens J. Holst ${ }^{2}$. Tine D. Clausen ${ }^{5}$. \\ Elisabeth R. Mathiesen ${ }^{6}$ Peter Damm ${ }^{7}$ • Filip K. Knop ${ }^{1,2,8}$ • Tina Vilsbøll ${ }^{1,8}$
}

Received: 18 October 2016 / Accepted: 27 February 2017 / Published online: 31 March 2017

(C) Springer-Verlag Berlin Heidelberg 2017

\begin{abstract}
Aims/hypothesis We investigated whether a reduced incretin effect, as observed in patients with type 2 diabetes, can be detected in high-risk individuals, such as women with prior gestational diabetes mellitus (pGDM).

Methods In this cross-sectional study, 102 women without diabetes with pGDM and 15 control participants without pGDM and with normal glucose tolerance (NGT) underwent a $4 \mathrm{~h} 75 \mathrm{~g}$ OGTT and an isoglycaemic i.v. glucose infusion (IIGI). Women with pGDM were classified as having NGT or prediabetes (impaired fasting glucose and/or impaired glucose tolerance). Insulin sensitivity was assessed using the Matsuda index and HOMA2IR and the incretin effect was calculated from insulin responses
\end{abstract}

Tina Vilsbøll

t.vilsboll@dadlnet.dk

1 Center for Diabetes Research, Gentofte Hospital, University of Copenhagen, Kildegårdsvej 28, DK-2900 Hellerup, Denmark

2 NNF Center for Basic Metabolic Research and Department of Biomedical Sciences, Faculty of Health and Medical Sciences, University of Copenhagen, Copenhagen, Denmark

3 Danish Diabetes Academy, Odense University Hospital, Odense, Denmark

4 Department of Gynecology and Obstetrics, Herlev Hospital, Herlev, Denmark

5 Department of Gynecology and Obstetrics, Nordsjællands Hospital, Hillerød, Denmark

6 Center for Pregnant Women with Diabetes, Department of Endocrinology, Rigshospitalet, Copenhagen, Denmark

7 Center for Pregnant Women with Diabetes, Department of Obstetrics, Rigshospitalet, Copenhagen, Denmark

8 Department of Clinical Medicine, Faculty of Health and Medical Sciences, University of Copenhagen, Copenhagen, Denmark during the study $\left(100 \% \times\left[\mathrm{AUC}_{\text {insulin,OGTT }}-\mathrm{AUC}_{\text {insulin,IIGI }}\right] /\right.$ $\mathrm{AUC}_{\text {insulin,OGTT }}$.

Results Sixty-three of the 102 women with pGDM (62\%) had prediabetes (median [interquartile range]: age, 38.3 [6.5] years; BMI, $32.1[5.8] \mathrm{kg} / \mathrm{m}^{2}$ ) and 39 women $(38 \%)$ had NGT (age, 39.5 [5.6] years; BMI, $31.0[6.7] \mathrm{kg} / \mathrm{m}^{2}$ ). Control participants $(n=15)$ were not significantly different from the pGDM group with regards to age (39.2 [7.4] years) and BMI $\left(28.8[9.2] \mathrm{kg} / \mathrm{m}^{2}\right)$. Compared with women with NGT and control participants, women with prediabetes had lower insulin sensitivity, as measured by the Matsuda index (3.0 [2.4] vs 5.0 [2.6] vs 1.5 [1.8], respectively; $p<0.001$ ). The incretin effect was $55.3 \%$ [27.8], 73.8\% [19.0] and 76.7\% [24.6] in women with prediabetes, women with normal glucose tolerance and control participants, respectively $(p<0.01)$.

Conclusion/interpretation Prediabetes was highly prevalent in women with pGDM, and alterations in the incretin effect were detected in this group before the development of type 2 diabetes. Trial registration: clinicaltrialsregister.eu 2012-001371-37-DK.

Keywords Gestational diabetes mellitus - Impaired glucose tolerance · Incretin effect - Isoglycaemic i.v. glucose infusion . Oral glucose tolerance test $\cdot$ Prediabetes $\cdot$ Type 2 diabetes

$\begin{array}{ll}\text { Abbreviations } \\ \text { FPG } & \text { Fasting plasma glucose } \\ \text { GCP } & \text { Good clinical practice } \\ \text { GDM } & \text { Gestational diabetes mellitus } \\ \text { GIGD } & \text { Gastrointestinal-mediated glucose disposal } \\ \text { GIP } & \text { Glucose-dependent insulinotropic polypeptide } \\ \text { GLP-1 } & \text { Glucagon-like peptide-1 } \\ \text { iAUC } & \text { Incremental AUC } \\ \text { IFG } & \text { Impaired fasting glucose } \\ \text { IGT } & \text { Impaired glucose tolerance }\end{array}$


IIGI Isoglycaemic i.v. glucose infusion

IQR Interquartile range

ISR Insulin secretory rate

NGT Normal glucose tolerance

pGDM Prior gestational diabetes mellitus

tAUC Total AUC

\section{Introduction}

Gestational diabetes mellitus (GDM) is defined as glucose intolerance detected during pregnancy [1]. In the majority of women with GDM, normal glucose tolerance (NGT) is reestablished after delivery $[2,3]$. Nevertheless, GDM represents one of the strongest predictors of development of type 2 diabetes later in life [4]. A review of 28 long-term follow-up studies revealed that up to $70 \%$ of women with prior GDM (pGDM) eventually progress to type 2 diabetes [5].

One of the first detectable deficiencies in the course of type 2 diabetes development is a reduced incretin effect $[6,7]$. The incretin effect is defined as the difference in insulin secretory responses elicited by oral and isoglycaemic i.v. glucose administration [8]. Studies have demonstrated that the impaired incretin effect in individuals with type 2 diabetes is caused mainly by impaired beta cell sensitivity to the insulinotropic incretin hormones (glucose-dependent insulinotropic polypeptide [GIP] and glucagon-like peptide-1 [GLP-1]) [9]. Kosinski et al observed that women with GDM exhibit a reduced incretin effect in the third trimester of pregnancy compared with women without GDM, and that this phenomenon was reversible alongside the restoration of NGT a few months after delivery [10]. Thus, women with pGDM appear to provide a unique opportunity for studying the metabolic changes preceding both prediabetes (impaired fasting glucose [IFG] and/or impaired glucose tolerance [IGT]) and type 2 diabetes. The objectives of this study were to characterise a group of women without diabetes (i.e. with NGT or prediabetes) with pGDM in relation to their incretin effect, as well as plasma levels of insulin, C-peptide, glucagon, GIP and GLP-1.

\section{Materials and methods}

Study design This study encompasses baseline assessments of women without diabetes but with pGDM. Participants were recruited as part of a randomised, placebo-controlled, doubleblinded intervention trial investigating the effect of a GLP-1 receptor agonist on glucose tolerance in women with pGDM [11]. The protocol was approved by the Danish Medicines Agency (EudraCT number: 2012-001371-27) and the Scientific-Ethical Committee of the Capital Region of Denmark (H-2-2012-073), and was registered with the Danish Data Protection Agency (01714 GEH-2012-024) and at www.clinicaltrialsregister.eu (2012-001371-37-DK). The study was carried out under the surveillance and guidance of the good clinical practice (GCP) unit at Copenhagen University Hospital, Copenhagen, Denmark, in compliance with The International Council for Harmonisation (ICH)GCP guidelines, and conducted in accordance with the Helsinki Declaration.

Study outcomes The primary outcome of this baseline assessment was incretin effect. Secondary endpoints included anthropometric and metabolic differences (waist:hip ratio, BP, fasting glucose, insulin resistance measured by HOMA2-IR and Matsuda index, dynamic responses of glucose, insulin, glucagon, GIP and GLP-1, insulin secretion rates [ISR], insulinogenic index, disposition index and gastrointestinalmediated glucose disposal [GIGD]).

Participants and recruitment Invitations to participate in the study were sent to all women $(n=2418)$ diagnosed with GDM within the last 10 years in three major obstetric departments in the Capital Region of Denmark. After initial screening over the telephone and/or e-mail, all eligible respondents (overweight women without diabetes, $n=121$ ) were invited for screening from September 2012 to August 2014. Women with screendetected type 2 diabetes $(n=5)$ were excluded and referred to their general practitioner according to standard recommendations. Eight women did not fulfil the inclusion criteria and three declined to participate; therefore, 105 women with pGDM were included in the study. Two women withdrew consent before participating in the isoglycaemic i.v. glucose infusion (IIGI) experiment and one woman was later excluded because of having monogenic diabetes, leaving 102 women for the crosssectional analysis. Based on their glucose tolerance status, as determined by OGTT, women with pGDM were divided into two groups: pGDM and NGT (pGDM-NGT), and pGDM and prediabetes (pGDM-prediabetes) in accordance with the WHO criteria [1]. Fifteen women with NGT and without pGDM and without diabetes among first-degree relatives were included prospectively in the trial as healthy control participants. The mean age, mean BMI and mean duration since pregnancy of the control group were matched with the pGDM group to reduce bias. All women gave written informed consent before screening.

Procedures For all women, a medical history was recorded and a physical examination was performed. All participants underwent an OGTT and an IIGI on separate days. On both occasions, the women were studied after an overnight fasting period $(10 \mathrm{~h})$. A cannula was inserted into a cubital vein and the hand was wrapped in a heating pad for collection of arterialised blood samples. On the day of the OGTT, participants ingested $75 \mathrm{~g}$ of water-free glucose dissolved in $300 \mathrm{ml}$ of water. On the IIGI day, an additional cannula was inserted into the contralateral cubital vein for infusion of sterile glucose 
(200 mg/ml, Frisenius Kabi, Bad Homburg, Germany) using an infusion pump (Model STC-508, Terumo, Tokyo, Japan). The infusion rate was continuously adjusted to replicate the glucose profile of the OGTT. On both days, blood samples were drawn at regular intervals from $15 \mathrm{~min}$ before until $240 \mathrm{~min}$ after ingestion of glucose for analyses of glucose, insulin, C-peptide, glucagon, GIP and GLP-1. Whole blood samples were distributed between chilled tubes containing EDTA plus a dipeptidyl-peptidase-4inhibitor (valine-pyrrolidide, a gift from Novo Nordisk, Bagsværd, Denmark) and serum tubes containing a clot activator. The latter samples were left to coagulate for $20 \mathrm{~min}$ at room temperature. Samples were stored at $-20^{\circ} \mathrm{C}$ and $-80^{\circ} \mathrm{C}$, respectively, until analysis.

Analyses Glucose was determined by the glucose oxidase method using a glucose analyser (Yellow Springs Instrument, 2300 STAT plus Analyzer, YSI, Yellow Springs, OH, USA). Insulin and C-peptide concentrations were measured using a two-sided electrochemiluminescence immunoassay (ADIVA Centaur XP, Siemens, Ballerup, Denmark). Glucagon was analysed using a C-terminal glucagon-specific antibody (code no. 4305; developed in house) [12]. Total GIP was measured using the C-terminal directed antiserum no. 80867 (developed in house), which reacts fully with intact GIP and the N-terminally truncated metabolite [13], and total GLP-1 was analysed using an antibody (code no. 89390; developed in house) specific for the amidated C-terminus of GLP-1 [14].

Calculations Mean concentrations of fasting values of glucose, insulin, GIP, GLP-1 and glucagon were calculated at $-15 \mathrm{~min}$, $-10 \mathrm{~min}$ and $0 \mathrm{~min}$. Minimum values for glucagon were expressed as the nadir. The AUC was calculated by the trapezoidal rule and expressed as either total AUC (tAUC) or incremental AUC (iAUC) from 0-240 min unless stated otherwise. The early insulin response was calculated as $\mathrm{iAUC}_{0-30}$, glucagon suppression as $\mathrm{tAUC}_{0-45}$ and $\mathrm{iAUC}_{0-45}$, and the early responses of GLP-1 and GIP as $\mathrm{iAUC}_{0-30}$, and the subsequent $2 \mathrm{~h}$ responses as $\mathrm{iAUC}_{0-120}$. ISR values were calculated by deconvolution of measured $\mathrm{C}$-peptide concentrations and application of population-based variables for C-peptide kinetics and expressed as pmol insulin secreted per min per $\mathrm{kg}$ body weight. Insulin resistance was evaluated using the computergenerated HOMA2-IR at www.dtu.ox.ac.uk (accessed on 11 January 2016) $[15,16]$. Insulin sensitivity during OGTT was calculated using the Matsuda index $[10,000 / \sqrt{ }($ fasting glucose $\times$ fasting insulin $) \times\left(\right.$ mean glucose ${ }_{0-120} \times$ mean insulin $\left.\left._{0-120}\right)\right]$ [17]. Insulinogenic indices (a measure of insulin release) were calculated as the ratio between $\Delta(0-30 \mathrm{~min})$ for insulin and glucose during both OGTT and IIGI [15]. Disposition index (insulinogenic index/HOMA2-IR) was used as an adjusted measure of beta cell function [18]. GIGD was calculated using the following formula: $100 \% \times$ (amount of glucose given during the OGTT - amount of glucose infusion during the IIGI)/amount of glucose given during the OGTT [19]. The incretin effect was calculated by relating the difference in integrated beta cell responses (iAUC for insulin or ISR $\times$ time) between stimulation of the gut (OGTT) or not (IIGI) to the response after the OGTT, multiplied by 100 to express the effect in per cent, i.e. $100 \% \times\left(\mathrm{AUC}_{\mathrm{OGTT}}-\mathrm{AUC}_{\mathrm{IIGI}}\right) / \mathrm{AUC}_{\text {OGTT }}[8]$.

Statistical methods Sample size was calculated based on the primary endpoint in the intervention study (change in glucose tolerance from baseline to week 52 as measured by the AUC for the glucose excursion following a $4 \mathrm{~h} 75$ g OGTT) [11]. Data are tabulated as median and interquartile range (IQR) or number and percentage. In the figures, data are presented as mean \pm SEM. We had only a few missing blood samples $(<0.7 \%)$ during the experimental days due to mechanical difficulties collecting blood from the cannula. The values for these missing samples were interpolated linearly. A $p$ value $<0.05$ was considered to be significant. The statistical analyses were performed using the statistical software GraphPad Prism 6.0 (GraphPad Software, La Jolla, CA, USA). Bartlett's test was used to test for normal distribution. Comparisons between the three groups were performed using the Kruskall-Wallis test with Dunn's correction for multiple comparisons for continuous variables, and the Mann-Whitney test when comparing two groups. Differences of categorical variables were analysed using $\chi^{2}$ with Bonferroni's test for multiple comparisons. Multivariate linear regression analysis was also performed: the incretin effect was used as a dependent variable and we included fasting plasma glucose (FPG), glucose response, fasting insulin level, insulin response, GIP response, GLP-1 response, glucagon response, the different indices of beta cell function, insulin resistance and the combined disposition index as prediction variables. In the initial models, we also adjusted for age, BMI and time since pregnancy. Subsequently, a stepwise model selection was performed using the MASS package in the statistical software R ( R 3.2.3, R Foundation for Statistical Computing, Vienna, Austria).

\section{Results}

Baseline characteristics Baseline characteristics of the control $(n=15)$, pGDM-NGT $(n=39)$ and pGDM-prediabetes $(n=63)$ groups are listed in Table 1. All women in the pGDMprediabetes group ( $62 \%$ of all pGDM) had IGT and only five of these women had concomitant IFG. The three groups were similar with regards to age, BMI and time since index pregnancy (the first pregnancy associated with GDM). Fasting glucose levels were higher in the pGDM-prediabetes group compared with the pGDM-NGT and control groups. $\mathrm{HbA}_{1 \mathrm{c}}$ was significantly higher in the pGDM-prediabetes group compared with the control group, but not compared with the pGDM-NGT group. $\mathrm{HbA}_{1 \mathrm{c}}$ was similar in the control and 
Table 1 Baseline characteristics and indices of beta cell dysfunction, insulin resistance and incretin effect

\begin{tabular}{|c|c|c|c|c|c|c|}
\hline Characteristic & Control & pGDM-NGT & pGDM-prediabetes & $p$ value $^{\mathrm{a}}$ & $p$ value $^{\mathrm{b}}$ & $p$ value $^{\mathrm{c}}$ \\
\hline Participants $(n)$ & 15 & 39 & 63 & & & \\
\hline Age (years) & $39.2(7.4)$ & $39.5(5.6)$ & $38.3(6.5)$ & $>0.999$ & 0.712 & 0.720 \\
\hline BMI $\left(\mathrm{kg} / \mathrm{m}^{2}\right)$ & $28.8(9.2)$ & $31.0(6.7)$ & $32.1(5.8)$ & $>0.999$ & 0.975 & 0.290 \\
\hline Fasting plasma glucose $(\mathrm{mmol} / \mathrm{l})$ & $4.9(0.6)$ & $5.2(0.5)$ & $5.5(0.8)$ & 0.136 & $<0.001$ & 0.021 \\
\hline $2 \mathrm{~h}$ glucose $(\mathrm{mmol} / \mathrm{l})^{\mathrm{d}}$ & $6.7(1.1)$ & $7.0(1.0)$ & $9.0(2.1)$ & $>0.999$ & $<0.001$ & $<0.001$ \\
\hline $\mathrm{HbA}_{1 \mathrm{c}}(\%)$ & $5.0(0.3)$ & $5.2(0.6)$ & $5.3(0.5)$ & 0.188 & 0.005 & 0.297 \\
\hline $\mathrm{HbA}_{1 \mathrm{c}}(\mathrm{mmol} / \mathrm{mol})$ & $31.3(3.2)$ & $33.4(7.1)$ & $34.3(6.2)$ & 0.322 & 0.012 & 0.292 \\
\hline Number of GDM pregnancies $(n)$ & NA & $1(0.0)$ & $1(0.0)$ & - & - & 0.448 \\
\hline Time since index pregnancy (years) & $5.1(5.9)$ & $4.7(3.7)$ & $4.7(4.2)$ & $>0.999$ & $>0.999$ & $>0.999$ \\
\hline Insulin treatment during GDM $(n)$ & NA & $4(10.3)$ & $17(27.0)$ & - & - & 0.042 \\
\hline Family history of diabetes $(n)$ & NA & $21(53.8)$ & $34(54.0)$ & - & - & 0.991 \\
\hline Use of hormonal contraceptive $(n)$ & $6(40.0)$ & $20(51.3)$ & $35(55.6)$ & $>0.999$ & $>0.999$ & $>0.999$ \\
\hline HOMA2-IR & $1.3(0.6)$ & $1.6(1.1)$ & $1.9(1.1)$ & 0.350 & 0.225 & $>0.999$ \\
\hline Matsuda index & $5.0(2.6)$ & $3.0(2.4)$ & $1.5(1.8)$ & 0.472 & $<0.001$ & $<0.001$ \\
\hline Insulinogenic index OGTT & $144(166)$ & $137(148)$ & $134(101)$ & $>0.999$ & 0.220 & 0.383 \\
\hline Disposition index OGTT & $104.7(125.0)$ & $88.0(99.9)$ & $74.1(71.0)$ & 0.360 & 0.021 & 0.412 \\
\hline Insulinogenic index IIGI & $45.3(59.4)$ & $49.7(50.0)$ & $45.1(46.0)$ & $>0.999$ & $>0.999$ & $>0.999$ \\
\hline Disposition index IIGI & $36.1(35.2)$ & $32.4(47.6)$ & $29.9(25.1)$ & $>0.999$ & 0.356 & $>0.999$ \\
\hline GIGD $(\%)$ & $60.9(19.9)$ & $60.0(17.1)$ & $46.0(20.2)$ & $>0.999$ & 0.001 & $<0.001$ \\
\hline Incretin effect (iAUC $\mathrm{insulin})(\%)$ & $76.7(24.6)$ & $73.8(19.0)$ & $55.3(27.8)$ & $>0.999$ & 0.002 & $<0.0001$ \\
\hline Incretin effect (iAUC ISR $)(\%)$ & $59.1(21.4)$ & $55.5(22.7)$ & $42.9(20.1)$ & 0.968 & $<0.001$ & $<0.001$ \\
\hline
\end{tabular}

Data are median (IQR) for continuous variables and $n(\%)$ for categorical variables

Continuous variables were analysed using the Kruskall-Wallis test with Dunn's correction for multiple comparisons test to obtain adjusted $p$ values. Differences of categorical variables were analysed by $\chi^{2}$ with Bonferroni's test for multiple comparisons when comparing three groups and Student's $t$ test when only comparing two groups. $p<0.05$ were considered significant

${ }^{\text {a }}$ Control vs pGDM-NGT

${ }^{\mathrm{b}}$ Control vs pGDM-prediabetes

${ }^{c}$ pGDM-NGT vs pGDM-prediabetes

${ }^{\mathrm{d}}$ Plasma glucose at $2 \mathrm{~h}$ during OGTT

NA, not applicable

the pGDM-NGT groups. There was no difference in the number of pregnancies with GDM between the pGDM groups, but more women were treated with insulin in at least one of their GDM pregnancies in the pGDM-prediabetes group $(n=17)$ compared with the pGDM-NGT group $(n=4)$.

Indices of beta cell function and insulin resistance Calculations from both the OGTT and IIGI days are listed in Table 1. Insulin resistance assessed by HOMA2-IR was similar in all three groups, whereas reduced insulin sensitivity as assessed by Matsuda index was found in the pGDMprediabetes group compared with both the pGDM-NGT and control groups. The insulinogenic index was similar in all three groups on both experimental days. Beta cell function corrected for insulin resistance (HOMA2-IR), i.e. the disposition index, showed a significant reduction in beta cell function on the OGTT day, but not on the IIGI day, in the pGDM-prediabetes group compared with control participants (Table 1).
Glucose and insulin Descriptive data and calculations from the OGTT and IIGI are listed in Table 2. Glucose curves during the OGTT and IIGI are shown in Fig. 1a-c. Compared with OGTTs, isoglycaemia during IIGIs was achieved in all three groups. Similar amounts of glucose were administered in the control and pGDM-NGT groups, whereas more glucose was needed to replicate the OGTT curve in the pGDM-prediabetes group, resulting in a lower GIGD in this group. Insulin responses are depicted in Fig. 1e-g, and the incretin effect calculated from the iAUC for insulin is illustrated in Fig. 1h. Compared with the other two groups, the control group had a lower insulin response during the OGTT (iAUC) and the pGDM-prediabetes group had a reduced early insulin response $\left(\mathrm{iAUC}_{0-30 \mathrm{~min}}\right)$. The same pattern was found for C-peptide responses (data not shown). The incretin effect calculated from the insulin iAUC was similar in the control group and the pGDM-NGT group, whereas the pGDM-prediabetes group exhibited a significantly lower incretin effect. The ISRs calculated 
Table 2 Data for glucose, insulin, ISR and glucagon from OGTT and IIGI

\begin{tabular}{|c|c|c|c|c|c|c|}
\hline Variable & Control & pGDM-NGT & pGDM-prediabetes & $p$ value $^{\mathrm{a}}$ & $p$ value $^{\mathrm{b}}$ & $p$ value $^{\mathrm{c}}$ \\
\hline \multicolumn{7}{|l|}{ Plasma glucose } \\
\hline \multicolumn{7}{|l|}{ OGTT } \\
\hline Peak (mmol/1) & $8.1(1.5)$ & $9.2(1.6)$ & $11.0(1.9)$ & 0.025 & $<0.001$ & $<0.001$ \\
\hline $\mathrm{tAUC}(\mathrm{mmol} / 1 \times \mathrm{min})$ & $1433(147)$ & $1502(168)$ & $1779(237)$ & 0.510 & $<0.001$ & $<0.001$ \\
\hline $\mathrm{iAUC}(\mathrm{mmol} / 1 \times \mathrm{min})$ & $203(77)$ & $252(122)$ & $528(185)$ & $>0.999$ & $<0.001$ & $<0.001$ \\
\hline \multicolumn{7}{|l|}{ IIGI } \\
\hline Peak (mmol/1) & $8.4(2.0)$ & $9.3(1.5)$ & $11.4(1.8)$ & 0.079 & $<0.001$ & $<0.001$ \\
\hline $\mathrm{tAUC}(\mathrm{mmol} / 1 \times \mathrm{min})$ & $1426(127)$ & $1514(194)$ & $1791(255)$ & 0.371 & $<0.001$ & $<0.001$ \\
\hline $\mathrm{iAUC}(\mathrm{mmol} / 1 \times \mathrm{min})$ & $218(73)$ & $276(140)$ & $481(214)$ & 0.362 & $<0.001$ & $<0.001$ \\
\hline \multicolumn{7}{|l|}{ Serum insulin } \\
\hline \multicolumn{7}{|l|}{ OGTT } \\
\hline Fasting (pmol/1) & $69.0(38.0)$ & $78.5(55.1)$ & $102(64.3)$ & $>0.999$ & 0.052 & 0.141 \\
\hline Peak (pmol/1) & $476(439)$ & $844(752)$ & $1009(750)$ & 0.199 & 0.236 & $>0.999$ \\
\hline tAUC $(\mathrm{nmol} / 1 \times \min )$ & $50.2(37.4)$ & $85.6(77.7)$ & $120(91.0)$ & 0.094 & 0.010 & $>0.999$ \\
\hline $\mathrm{iAUC}(\mathrm{nmol} / 1 \times \mathrm{min})$ & $38.2(22.1)$ & $62.5(59.0)$ & $91.9(8.0)$ & 0.041 & 0.004 & $>0.999$ \\
\hline $\mathrm{iAUC}_{0-30}(\mathrm{nmol} / 1 \times \mathrm{min})$ & $5.1(2.6)$ & $5.5(4.5)$ & $4.5(4.1)$ & $>0.999$ & 0.094 & 0.012 \\
\hline \multicolumn{7}{|l|}{ IIGI } \\
\hline Fasting (pmol/1) & $80.5(60.9)$ & $83.0(64.6)$ & $110(66.9)$ & $>0.999$ & 0.612 & 0.498 \\
\hline $\mathrm{tAUC}(\mathrm{nmol} / 1 \times \min )$ & $33.3(18.3)$ & $32.2(32.3)$ & $61.4(49.4)$ & 0.988 & 0.006 & 0.010 \\
\hline $\mathrm{iAUC}(\mathrm{nmol} / 1 \times \mathrm{min})$ & $7.4(13.2)$ & $14.4(23.3)$ & $34.3(33.0)$ & 0.430 & $<0.001$ & 0.001 \\
\hline $\mathrm{iAUC}_{0-30}(\mathrm{nmol} / 1 \times \mathrm{min})$ & $1.4(1.5)$ & $1.9(1.5)$ & $0.7(0.9)$ & $>0.999$ & 0.004 & $<0.001$ \\
\hline \multicolumn{7}{|l|}{ ISR } \\
\hline \multicolumn{7}{|l|}{ OGTT } \\
\hline Peak (pmol min ${ }^{-1} \mathrm{~kg}^{-1}$ ) & $7.1(4.5)$ & $10.5(2.2)$ & $10.5(3.3)$ & 0.002 & 0.003 & $>0.999$ \\
\hline $\mathrm{tAUC}(\mathrm{pmol} \times \mathrm{kg})$ & $904(480)$ & $1244(555)$ & $1431(564)$ & 0.018 & $<0.001$ & 0.036 \\
\hline iAUC $($ pmol × kg) & $532(276)$ & $838(388)$ & $1028(424)$ & 0.012 & $<0.001$ & 0.008 \\
\hline \multicolumn{7}{|l|}{ IIGI } \\
\hline Peak (pmol min ${ }^{-1} \mathrm{~kg}^{-1}$ ) & $3.7(2.3)$ & $5.8(3.8)$ & $7.4(3.9)$ & 0.025 & $<0.001$ & 0.076 \\
\hline $\mathrm{tAUC}(\mathrm{pmol} \times \mathrm{kg})$ & $591(370)$ & $739(465)$ & $1047(525)$ & 0.163 & $<0.001$ & 0.001 \\
\hline $\mathrm{iAUC}(\mathrm{pmol} \times \mathrm{kg})$ & $216(142)$ & $378(233)$ & 509 (396) & 0.043 & $<0.001$ & $<0.001$ \\
\hline \multicolumn{7}{|l|}{ Glucagon } \\
\hline \multicolumn{7}{|l|}{ OGTT } \\
\hline Fasting, (pmol/1) & $5.3(4.3)$ & $6.0(2.7)$ & $6.3(2.7)$ & $>0.999$ & $>0.999$ & $>0.999$ \\
\hline Nadir (pmol/l) & $1.0(3.0)$ & $2.0(3.0)$ & $2.0(2.0)$ & $>0.999$ & $>0.999$ & $>0.999$ \\
\hline $\mathrm{tAUC}_{0-45}(\mathrm{pmol} / 1 \times \mathrm{min})$ & $205(155)$ & $258(85)$ & $270(110)$ & $>0.999$ & 0.347 & $>0.999$ \\
\hline $\mathrm{iAUC}_{0-45}(\mathrm{pmol} / 1 \times \mathrm{min})$ & $-54(68)$ & $-18(85)$ & $-7(61)$ & 0.288 & 0.004 & 0.723 \\
\hline \multicolumn{7}{|l|}{ IIGI } \\
\hline Fasting (pmol/1) & $6.3(4.0)$ & $6.3(2.4)$ & $6.5(2.3)$ & $>0.999$ & $>0.999$ & $>0.999$ \\
\hline Nadir (pmol/1) & $2.0(2.9)$ & $1.0(2.9)$ & $2.0(2.9)$ & $>0.999$ & $>0.999$ & $>0.999$ \\
\hline $\mathrm{tAUC}_{0-45}(\mathrm{pmol} / 1 \times \mathrm{min})$ & $245(117)$ & $223(94)$ & $253(103)$ & $>0.999$ & $>0.999$ & $>0.999$ \\
\hline $\mathrm{iAUC}_{0-45}(\mathrm{pmol} / 1 \times \mathrm{min})$ & $-84(86)$ & $-54(50)$ & $-45(42)$ & 0.439 & 0.051 & $>0.999$ \\
\hline
\end{tabular}

Data are median (IQR)

Variables were analysed using the Kruskall-Wallis test with Dunn's correction for multiple comparisons test to obtain adjusted $p$ values

${ }^{\text {a }}$ Control vs pGDM-NGT

${ }^{\mathrm{b}}$ Control vs pGDM-prediabetes

${ }^{\mathrm{c}}$ pGDM-NGT vs pGDM-prediabetes 

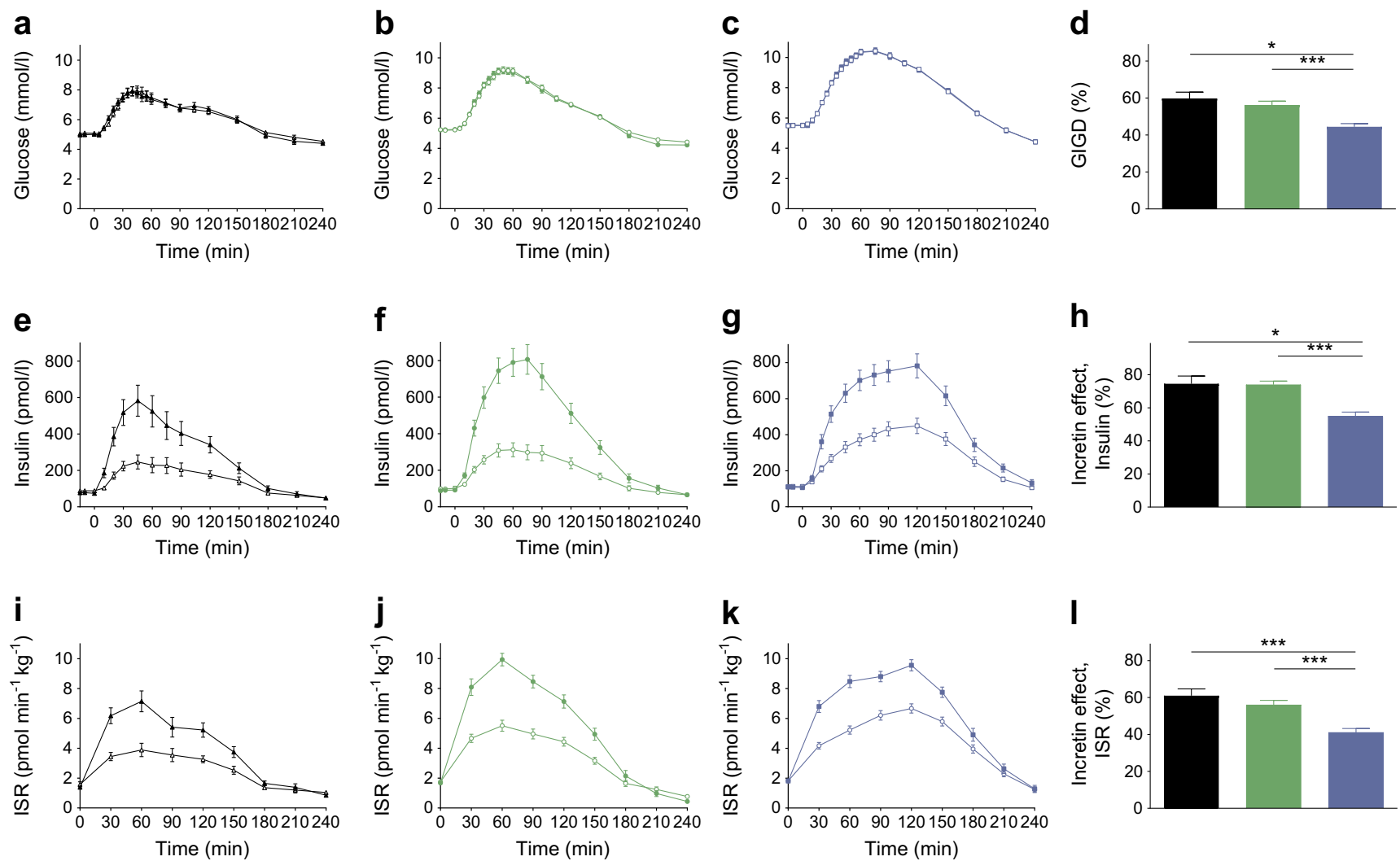

Fig. 1 Glucose, insulin and ISR. (a-c) Glucose excursions. (d) GIGD. (e-g) Insulin excursions. (h) Incretin effect calculated from incremental insulin responses. (i-k) ISR calculated from C-peptide excursions. (l)

from C-peptide responses are depicted in Fig. 1i-k. As illustrated, the control and pGDM-NGT groups peaked after $60 \mathrm{~min}$, whereas the pGDM-prediabetes group peaked after $120 \mathrm{~min}$. The incretin effect based on the ISR iAUC is shown in Fig. 11. This was similar in the control and pGDM-NGT groups, whereas the pGDM-prediabetes group exhibited a significantly lower incretin effect.

Glucagon, GIP and GLP-1 The responses of glucagon, GIP and GLP-1 on the OGTT and IIGI days are depicted in Fig. 2. Detailed descriptive data are listed for glucagon in Table 2, and for GIP and GLP-1 in Table 3. Fasting and nadir values for glucagon responses were similar in all groups on both days. The control group initiated the suppression of glucagon after $10 \mathrm{~min}$, whereas both pGDM groups exhibited a delayed suppression resulting in a significantly higher iAUC (0-45 $\mathrm{min})$ during the OGTT compared with the IIGI in these groups (pGDM-NGT, $p=0.019$; pGDM-prediabetes, $p<0.001$ ). Fasting, peak, tAUC and iAUC values for GIP, GLP-1 and GLP-1 (0-30 min) during the OGTT were similar in the three groups. The GLP-1 (0-120 $\mathrm{min})$ response was slightly reduced in the pGDM-prediabetes group compared with the control group and the pGDM-NGT group. The GIP (0-120 min) response was also slightly reduced in the pGDM-prediabetes group compared with the pGDM-NGT group.
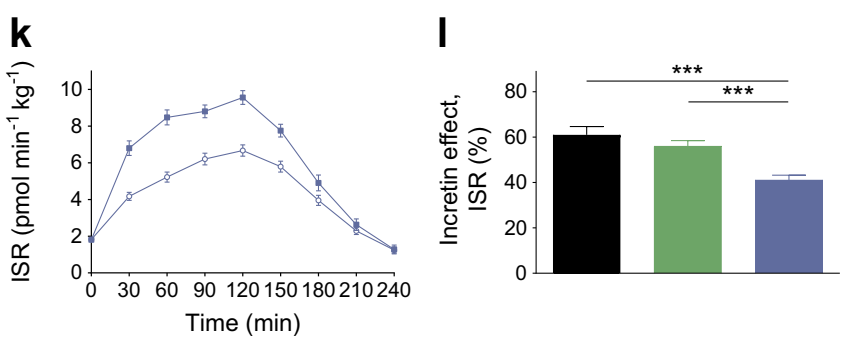

Incretin effect was calculated from ISR. Filled symbols, OGTT day; open symbols, IIGI day. Black, control; green, pGDM-NGT; blue, pGDMprediabetes. Data are mean \pm SEM. $* p<0.05, * * * p<0.001$

Multivariate linear regression analysis We tested the six possible models based on the different insulin resistance measures (HOMA2-IR and Matsuda index) combined with the insulinogenic index based on either OGTT or IIGI, or the disposition index based on either OGTT or IIGI. The stepwise regression of the six models resulted in three alternative models describing the incretin effect (model A: incretin effect $=\mathrm{BMI}+\mathrm{FPG}+$ glucose response + fasting insulin + GLP1 response; model B: incretin effect $=$ FPG + glucose response + disposition index IIGI + GLP1; and model C: incretin effect $=$ FPG + glucose response + fasting insulin + insulinogenic index IIGI + insulin response + GLP1 response). Common for all models was the inclusion of the FPG, the glucose response and the GLP-1 response as significant contributors. All oral indices of beta cell function and insulin resistance were excluded, whereas the indices based on i.v. glucose were retained in the models. All models reached high significance $(p<0.001)$ and the models were able to describe between $40 \%$ and $47 \%$ of the variation in the incretin effect (model A: $\left.R^{2}=0.396 ; \mathrm{B}: R^{2}=0.401 ; \mathrm{C}: R^{2}=0.468\right)$.

\section{Discussion}

Here, we show the presence of prediabetes $(62 \%)$ in a cohort of otherwise healthy women with pGDM, and that women with 

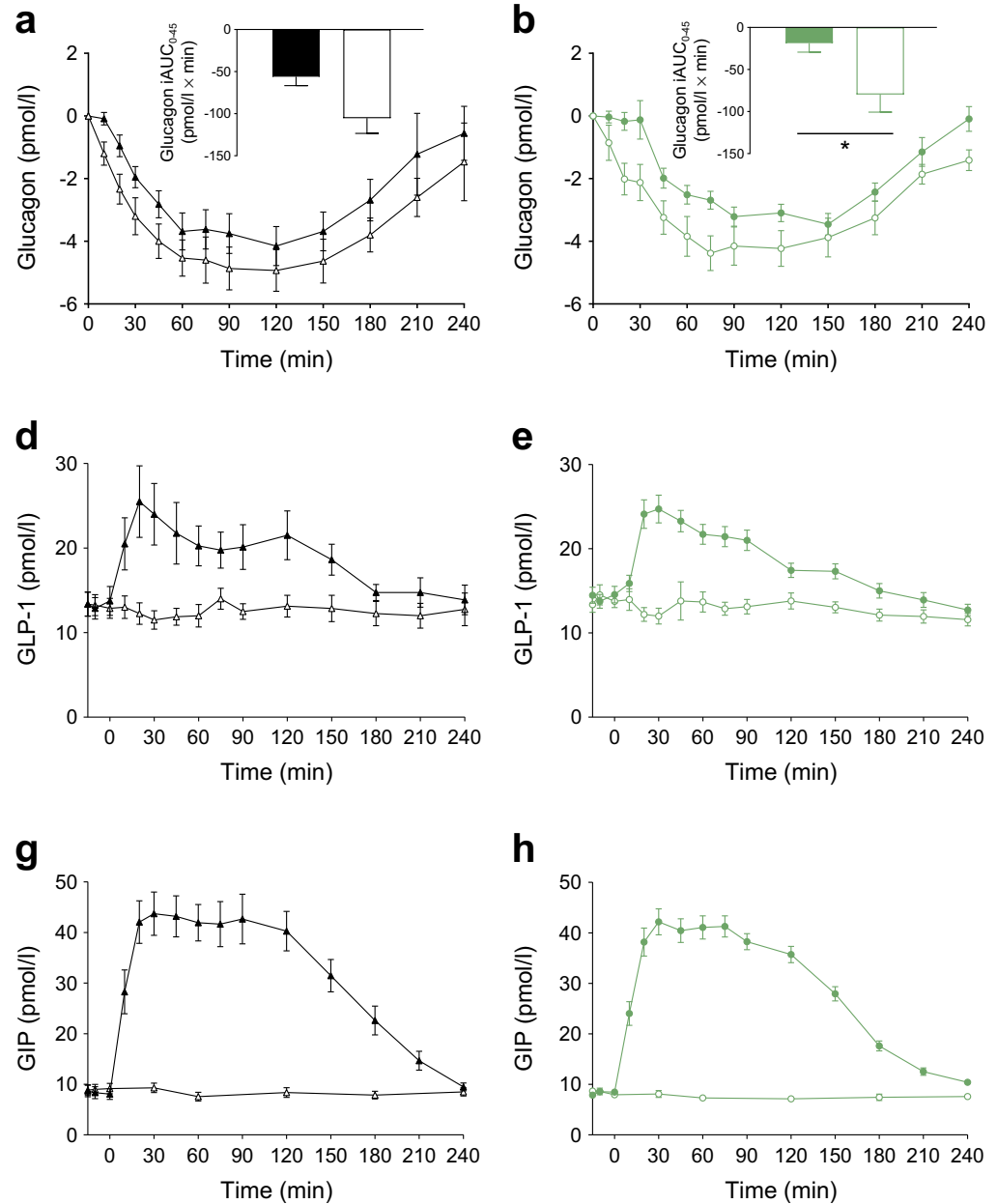
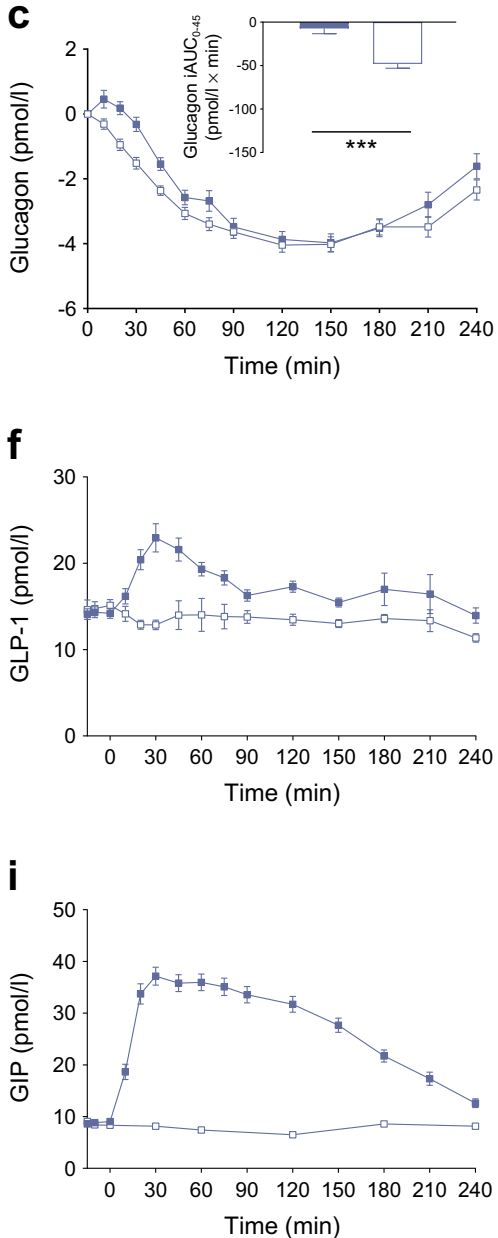

Fig. 2 Glucagon, GLP-1 and GIP responses. (a-c) Glucagon excursion and (inset) glucagon iAUC ${ }_{0-45}$. (d-f) Total GLP-1 excursion. (g-i) Total GIP response. Filled symbols, OGTT day; open symbols, IIGI day. Black, control; green, pGDM-NGT; blue, pGDM-prediabetes. Data are mean \pm SEM. $* p<0.05, * * * p<0.001$

therefore appears to be representative of the GDM population in Denmark. Our analyses of hormone responses are based on frequent blood sampling throughout the $240 \mathrm{~min}$ OGTT and IIGI, which strengthens the accuracy of the AUC calculations. Finally, we used well-documented assays for analysing plasma samples $[13,20]$. Limitations include the a priori exclusion of women diagnosed with overt type 2 diabetes, possibly creating a cohort that is less predisposed to the development of type 2 diabetes. Conversely, we excluded women with normal weight, which should have the opposite effect on the cumulative risk for future type 2 diabetes [2].

It is known that the incretin effect is reduced in the third trimester of pregnancy in women with GDM and that this decrease is fully reversed within a few months after delivery, alongside normalisation of glucose tolerance [10]. The incretin effect has, however, not been studied in women with pGDM until now. It has been studied in adolescents with prediabetes who, consistent with our results, have a decreased incretin effect [21]. The women in our study population were obese, a factor which has been shown to reduce the incretin effect in men with 
Table 3 Data for GLP-1 and GIP from OGTT

\begin{tabular}{|c|c|c|c|c|c|c|}
\hline Variable & Control & pGDM-NGT & pGDM-prediabetes & $p$ value $^{\mathrm{a}}$ & $p$ value $^{\mathrm{b}}$ & $p$ value $\mathrm{e}^{\mathrm{c}}$ \\
\hline \multicolumn{7}{|l|}{ GLP-1 } \\
\hline Fasting (pmol/1) & $11.0(5.4)$ & $12.3(5.3)$ & $13.0(5.5)$ & $>0.999$ & 0.416 & $>0.999$ \\
\hline Peak (pmol/l) & $24.0(14.0)$ & $26.0(12.0)$ & $24.0(10.0)$ & $>0.999$ & $>0.999$ & $>0.999$ \\
\hline tAUC $(\mathrm{pmol} / 1 \times \min )$ & $3889(1898)$ & $3875(1516)$ & $3779(1346)$ & $>0.999$ & $>0.999$ & $>0.999$ \\
\hline iAUC $(\mathrm{pmol} / 1 \times \mathrm{min})$ & $1259(851)$ & 981 (1309) & $696(866)$ & 0.710 & 0.056 & 0.363 \\
\hline $\mathrm{iAUC}_{0-30}(\mathrm{pmol} / \mathrm{l} \times \mathrm{min})$ & $132(151)$ & $150(118)$ & $90(170)$ & $>0.999$ & 0.316 & 0.327 \\
\hline $\mathrm{iAUC}_{0-120}(\mathrm{pmol} / 1 \times \mathrm{min})$ & $819(510)$ & $759(728)$ & $446(604)$ & $>0.999$ & 0.016 & 0.038 \\
\hline \multicolumn{7}{|l|}{ GIP } \\
\hline Fasting (pmol/l) & $7.7(3.0)$ & $8.0(3.7)$ & $8.0(3.6)$ & $>0.999$ & $>0.999$ & $>0.999$ \\
\hline Peak (pmol/l) & $43.0(29.0)$ & $43.0(29.0)$ & $40.0(16.0)$ & $>0.999$ & 0.717 & 0.505 \\
\hline $\mathrm{tAUC}(\mathrm{pmol} / 1 \times \mathrm{min})$ & $7268(4642)$ & $6672(2130)$ & $6253(2493)$ & $>0.999$ & 0.542 & $>0.999$ \\
\hline $\mathrm{iAUC}(\mathrm{pmol} / 1 \times \mathrm{min})$ & $5078(2303)$ & $4783(2286)$ & 4354 (2358) & $>0.999$ & 0.202 & 0.517 \\
\hline $\mathrm{iAUC}_{0-30}(\mathrm{pmol} / 1 \times \mathrm{min})$ & $643(393)$ & $592(305)$ & $497(360)$ & $>0.999$ & 0.059 & 0.099 \\
\hline $\mathrm{iAUC}_{0-120}(\mathrm{pmol} / 1 \times \mathrm{min})$ & $3378(2438)$ & $3273(1700)$ & $2790(1825)$ & $>0.999$ & 0.065 & 0.031 \\
\hline
\end{tabular}

Values are median (IQR)

Variables were analysed using the Kruskall-Wallis test with Dunn's correction for multiple comparisons test to obtain adjusted $p$ values. $p$ values $<0.05$ were considered significant

${ }^{\text {a }}$ Control vs pGDM-NGT

${ }^{\mathrm{b}}$ Control vs pGDM-prediabetes

${ }^{\mathrm{c}}$ pGDM-NGT vs pGDM-prediabetes

NGT and type 2 diabetes [22], and which, therefore corroborates our results. However, we did not find a reduced incretin effect in our NGT group, possibly because our cohort was about 20 years younger and six BMI points leaner than the men studied by Knop et al [22]. Factors other than the incretin effect might affect glucose disposal differently during oral and i.v. glucose administration; these factors include neural reflexes, activation of afferent nerves in the intestinal mucosa, differences in glucagon secretion, first-pass hepatic uptake of glucose, differences in portal and venous blood glucose concentrations, direct nutrient effect and possibly presently unknown factors. The GIGD takes all of these factors into account [23]. We demonstrated a preserved GIGD in the control group and pGDM-NGT group, and a reduction in the pGDM-prediabetes group, which is similar to our findings for the incretin effect. This suggests that the incretin effect represents a major contributor to the GIGD in our population.

Although within the normal range, higher fasting glucose was found in the pGDM-prediabetes group compared with the pGDM-NGT and control groups. Only $8 \%$ of the women in the pGDM-prediabetes group had combined IFG and IGT, while the vast majority had isolated IGT. No differences in insulin resistance as measured by HOMA-IR were found between the groups. Since fasting insulin was similar in all three groups and fasting glucose followed the same pattern, the HOMA-IR indices based on fasting levels were not affected in our population. In contrast, the pGDM-prediabetes group had reduced insulin sensitivity as measured by the Matsuda index (based on the differences in dynamics of glucose and insulin during the OGTT). Equally, the immediate increase in ISR after glucose ingestion seemed to be weakened in the pGDM-prediabetes group with time to peak being reached at 120 min compared with $60 \mathrm{~min}$ in the two NGT groups, implying that this group has some degree of beta cell dysfunction. Disposition index (a surrogate measure of beta cell function) was also reduced in the pGDM-prediabetes group during the OGTT, which may reflect a decreased ability to adequately amplify insulin secretion to oral glucose stimulation. Collectively, this suggests that, at least temporarily, women with pGDM are able to retain NGT because their beta cells produce large amounts of insulin, but at some point the insulin production is not adequate and IGT develops.

People with type 2 diabetes are known to have elevated fasting glucagon and exhibit decreased suppression of glucagon after glucose ingestion [24]. We did not find any alterations in fasting or nadir glucagon values in the pGDM groups on either of the test days, but we did find a delay (up to $30 \mathrm{~min}$ ) in the immediate suppression of glucagon on the OGTT day compared with the IIGI day in both pGDM groups. In contrast, the control participants were capable of immediate suppression of glucagon after administration of glucose, which is central to the maintenance of normal glucose excursions after a meal or an oral glucose load in healthy individuals. Normal fasting glucagon and delayed suppression during an OGTT have previously been found in both lean and obese individuals with prediabetes [25]. However, these participants, in contrast to ours, also had 
increased nadir glucagon values on the OGTT day compared with control participants. A study by Mitrakou et al [26] included individuals with combined IFG/IGT. The five participants in our study with combined IFG/IGT also had elevated fasting glucagon, an initial increase in glucagon, delayed suppression and increased nadir glucagon values during the OGTT (data not shown). However, the majority of individuals in the prediabetes group in our study had solely IGT and appeared to be healthier than both the IGT group investigated by Mitrakou et al [26] and individuals with type 2 diabetes [24] with elevated FPG and fasting glucagon, because, during their participation, they only appeared to be experiencing masked beta cell dysfunction and mild alpha cell dysfunction.

We analysed total GLP-1 as a measure of GLP-1 secretion and focused on the early phases of the response to relate our results to previously reported data. The GLP-1 (0-120 min) response was reduced in the pGDM-prediabetes group compared with both NGT groups, which is consistent with the findings of Færch et al [25] and Laakso et al [27]. No reduction was found in the early $(0-30 \mathrm{~min})$ response in contrast to findings by Færch et al [25] and Forbes et al [28]. Furthermore, in contrast to Laakso et al [27], we found no changes in the full GLP-1 $(0-240 \mathrm{~min})$ response. The GIP $(0-120 \mathrm{~min})$ response was reduced during the OGTT in the pGDM-prediabetes group compared with the pGDM-NGT group. No differences were found in the early $(0-30 \mathrm{~min})$ response or the full GIP $(0-240)$ response. Meier et al investigated 20 women with pGDM and found that the $0-120$ min GIP response during an OGTT was similar to a control group [29]. This is in agreement with our results for the pGDM-NGT group. However, the women included in the trial by Meier et al generally had fewer metabolic alterations and the majority had NGT, which could explain the minor differences between the GIP and GLP-1 responses when compared with control participants without pGDM [29]. In some studies, individuals with type 2 diabetes have slightly, but not clinically relevant, reduced GLP-1 levels [30], and it is generally acknowledged that the impaired incretin effect in type 2 diabetes, is primarily caused by an impaired insulinotropic response to GIP and GLP-1. As we will follow this cohort of women with pGDM prospectively for 5 years, we have a unique opportunity to observe if the women with reduced GLP-1 responses will go on to develop type 2 diabetes [11].

Preventing the development of type 2 diabetes in high-risk individuals remains a hot, and clinically relevant, topic. The ADA advises all women with pGDM to be screened for type 2 diabetes at 6-12 weeks after delivery and every second to third year thereafter [31]. Additionally, in the USA, lifestyle interventions and/or metformin are recommended for women with pGDM and prediabetes to prevent type 2 diabetes, which is in contrast to most European countries, where only lifestyle interventions are recommended [31,32]. As aforementioned, the cohort of women with pGDM in the present study is currently enrolled in a randomised clinical trial with the aim to shed light on the effect of treating these high-risk individuals with a GLP-1 analogue [11].

In conclusion, we characterised a group of women without diabetes but with pGDM and found that prediabetes was highly prevalent in these individuals. The incretin effect and GIGD were unchanged in women with pGDM and NGT, but were decreased in women with pGDM and prediabetes. The reduced incretin effect may be caused by a combination of decreased sensitivity to and secretion of incretins.

Acknowledgements The authors are grateful to the participating women and for laboratory assistance from: N. Kjeldsen, J. Purtoft, S. M. Schmidt and I. A. Nachar at Center for Diabetes Research, Gentofte Hospital, University of Copenhagen, Hellerup, Denmark; L. Thielsen and L. Albæk from the Novo Nordisk Foundation (NNF) Center for Basic Metabolic Research and Department of Biomedical Sciences, Faculty of Health and Medical Sciences, University of Copenhagen, Denmark; and Novo Nordisk, Bagsværd, Denmark for the supply of valine-pyrrolidide. Part of this work has been presented as an abstract at the Scientific Sessions of the ADA in San Francisco, June 2014, and Boston, June 2015, and at the Annual Meeting of the EASD in Vienna, September 2014, and Stockholm, September 2015.

Data availability The datasets generated and analysed during the current study are available from the corresponding author on reasonable request.

Funding The study was initiated by the authors and supported as an investigator-initiated study by unrestricted grants from Novo Nordisk, the Novo Nordisk Foundation and the Danish Diabetes Academy. Research support was received from the AP Moeller Foundation and Danielsen Foundation. The study sponsor (Novo Nordisk) was involved in the design of the study; but not the collection, analysis, and interpretation of data; neither writing the report; nor the decision to submit the report for publication.

Duality of interest SF, LV, CA, ESA, EB, JIB, TDC, JAS, ERM declare that there is no duality of interest associated with this manuscript. JJH is on the advisory panel of, consultant for, in the speaker bureau of and/or has received research support from AstraZeneca, GlaxoSmithKline, Hamni, Intarcia, Merck Sharp \& Dohme, Novartis, Novo Nordisk, Sanofi and Zealand Pharma. PD is on the speaker bureau for Novo Nordisk and is participating in a multinational study with Novo Nordisk. FKK is on the advisory panel of, consultant for, in the speaker bureau of and/or has received research support from Amgen, AstraZeneca, Boehringer Ingelheim, Eli Lilly, Fractyl, Gubra, Novo Nordisk, Merck Sharp \& Dohme, Sanofi and Zealand Pharma. TV is on the advisory panel for, consultant for, in the speaker bureau of and/or has received research support from Amgen, AstraZeneca, Boehringer Ingelheim, Bristol-Myers Squibb, Eli Lilly, Merck Sharp \& Dohme, Novartis, Novo Nordisk, Sanofi and Takeda.

Contribution statement All authors critically revised the manuscript for important intellectual content as well as approved the final version. SF and LV made contributions to conception and design, acquisition of data, and analysis and interpretation of data, and are responsible for the integrity of the data. CA, ESA and EB contributed to acquisition of data, and analysis and interpretation of data. JJH contributed to analysis and interpretation of data. JAS, TDC, ERM and PD were responsible for recruitment of patients and contributed to interpretation of data. JIB contributed to interpretation of data. FKK contributed to the design of the study and interpretation of data. TV is the guarantor of this work and made substantial contributions to conception and design, acquisition of data, analysis and interpretation of data, obtained funding and is responsible for the integrity of the data. 


\section{References}

1. World Health Organization, International Diabetes Federation (2006) Definition and diagnosis of diabetes mellitus and intermediate hyperglycemia: report of a WHO/IDF consultation. WHO, Geneva

2. Lauenborg J, Hansen T, Jensen DM et al (2004) Increasing incidence of diabetes after gestational diabetes: a long-term follow-up in a Danish population. Diabetes Care 27:1194-1199

3. Kitzmiller JL, Dang-Kilduff L, Taslimi MM (2007) Gestational diabetes after delivery. Short-term management and long-term risks. Diabetes Care 30(Suppl 2):S225-S235

4. Benhalima K, Mathieu C, Damm P et al (2015) A proposal for the use of uniform diagnostic criteria for gestational diabetes in Europe: an opinion paper by the European Board \& College of Obstetrics and Gynaecology (EBCOG). Diabetologia 58:1422-1429

5. Kim C, Newton KM, Knopp RH (2002) Gestational diabetes and the incidence of type 2 diabetes: a systematic review. Diabetes Care 25:1862-1868

6. Fernández-Castañer M, Biarnés J, Camps I, Ripollés J, Gómez N, Soler J (1996) Beta-cell dysfunction in first-degree relatives of patients with non-insulin-dependent diabetes mellitus. Diabet Med J 13:953-959

7. Kahn SE, Cooper ME, Del Prato S (2014) Pathophysiology and treatment of type 2 diabetes: perspectives on the past, present, and future. Lancet 383:1068-1083

8. Nauck M, Stöckmann F, Ebert R, Creutzfeldt W (1986) Reduced incretin effect in type 2 (non-insulin-dependent) diabetes. Diabetologia 29:46-52

9. Vilsbøll T, Krarup T, Madsbad S, Holst JJ (2002) Defective amplification of the late phase insulin response to glucose by GIP in obese type II diabetic patients. Diabetologia 45:1111-1119

10. Kosinski M, Knop FK, Vedtofte L et al (2013) Postpartum reversibility of impaired incretin effect in gestational diabetes mellitus. Regul Pept 186:104-107

11. Foghsgaard S, Vedtofte L, Mathiesen ER et al (2013) The effect of a glucagon-like peptide-1 receptor agonist on glucose tolerance in women with previous gestational diabetes mellitus: protocol for an investigator-initiated, randomised, placebo-controlled, doubleblinded, parallel intervention trial. BMJ Open 3:e003834. doi:10. 1136/bmjopen-2013-003834

12. Wewer Albrechtsen NJ, Hartmann B, Veedfald S et al (2014) Hyperglucagonaemia analysed by glucagon sandwich ELISA: non-specific interference or truly elevated levels? Diabetologia 57:1919-1926

13. Lindgren $\mathrm{O}$, Carr RD, Deacon CF et al (2011) Incretin hormone and insulin responses to oral versus intravenous lipid administration in humans. J Clin Endocrinol Metab 96:2519-2524

14. Orskov C, Wettergren A, Holst JJ (1993) Biological effects and metabolic rates of glucagonlike peptide-1 7-36 amide and glucagonlike peptide-1 7-37 in healthy subjects are indistinguishable. Diabetes 42:658-661

15. Matthews DR, Hosker JP, Rudenski AS, Naylor BA, Treacher DF, Turner RC (1985) Homeostasis model assessment: insulin resistance and beta-cell function from fasting plasma glucose and insulin concentrations in man. Diabetologia 28:412-419

16. Levy JC, Matthews DR, Hermans MP (1998) Correct homeostasis model assessment (HOMA) evaluation uses the computer program. Diabetes Care 21:2191-2192
17. Matsuda M, DeFronzo RA (1999) Insulin sensitivity indices obtained from oral glucose tolerance testing: comparison with the euglycemic insulin clamp. Diabetes Care 22:1462-1470

18. Turner RC, Holman RR, Matthews D, Hockaday TD, Peto J (1979) Insulin deficiency and insulin resistance interaction in diabetes: estimation of their relative contribution by feedback analysis from basal plasma insulin and glucose concentrations. Metabolism 28:1086-1096

19. Hare KJ, Vilsbøll T, Holst JJ, Knop FK (2010) Inappropriate glucagon response after oral compared with isoglycemic intravenous glucose administration in patients with type 1 diabetes. Am J Physiol Endocrinol Metab 298:E832-E837

20. Orskov C, Rabenhøj L, Wettergren A, Kofod H, Holst JJ (1994) Tissue and plasma concentrations of amidated and glycineextended glucagon-like peptide I in humans. Diabetes 43:535-539

21. Michaliszyn SF, Mari A, Lee S et al (2014) $\beta$-Cell function, incretin effect, and incretin hormones in obese youth along the span of glucose tolerance from normal to prediabetes to type 2 diabetes. Diabetes 63:3846-3855

22. Knop FK, Aaboe K, Vilsbøll T et al (2012) Impaired incretin effect and fasting hyperglucagonaemia characterizing type 2 diabetic subjects are early signs of dysmetabolism in obesity. Diabetes Obes Metab 14:500-510

23. Holst JJ, Knop FK, Vilsbøll T, Krarup T, Madsbad S (2011) Loss of incretin effect is a specific, important, and early characteristic of type 2 diabetes. Diabetes Care 34(Suppl 2):S251-S257

24. Bagger JI, Knop FK, Lund A, Holst JJ, Vilsbøll T (2014) Glucagon responses to increasing oral loads of glucose and corresponding isoglycaemic intravenous glucose infusions in patients with type 2 diabetes and healthy individuals. Diabetologia 57:1720-1725

25. Færch K, Torekov SS, Vistisen D et al (2015) GLP-1 response to oral glucose is reduced in prediabetes, screen-detected type 2 diabetes, and obesity and influenced by sex: the ADDITION-PRO study. Diabetes 64:2513-2525

26. Mitrakou A, Kelley D, Mokan M et al (1992) Role of reduced suppression of glucose production and diminished early insulin release in impaired glucose tolerance. N Engl J Med 326:22-29

27. Laakso M, Zilinskaite J, Hansen $T$ et al (2008) Insulin sensitivity, insulin release and glucagon-like peptide-1 levels in persons with impaired fasting glucose and/or impaired glucose tolerance in the EUGENE2 study. Diabetologia 51:502-511

28. Forbes S, Godsland IF, Taylor-Robinson SD et al (2013) A history of previous gestational diabetes mellitus is associated with adverse changes in insulin secretion and VLDL metabolism independently of increased intrahepatocellular lipid. Diabetologia 56:2021-2033

29. Meier JJ, Gallwitz B, Askenas M et al (2005) Secretion of incretin hormones and the insulinotropic effect of gastric inhibitory polypeptide in women with a history of gestational diabetes. Diabetologia 48 : 1872-1881

30. Calanna S, Christensen M, Holst JJ et al (2013) Secretion of glucagon-like peptide- 1 in patients with type 2 diabetes mellitus: systematic review and meta-analyses of clinical studies. Diabetologia 56:965-972

31. American Diabetes Association (2016) II. Classification and diagnosis of diabetes. Diabetes Care 39(Suppl 2):S137-S145

32. Metzger BE, Coustan DR (1998) Summary and recommendations of the Fourth International Workshop-Conference on Gestational Diabetes Mellitus. The Organizing Committee. Diabetes Care 21(Suppl 2):B161-B167 